\title{
A GENERALIZED UPPER AND LOWER SOLUTIONS METHOD FOR NONLINEAR SECOND ORDER ORDINARY DIFFERENTIAL EQUATIONS ${ }^{1}$
}

\author{
JUAN J. NIETO ${ }^{2}$ \\ Departamento de Análisis Matemático \\ Facultad de Matemáticas \\ Universidad de Santiago de Compostela \\ SPAIN \\ ALBERTO CABADA ${ }^{2}$ \\ Departamento de Matemática Aplicada \\ Facultad de Matemáticas \\ Universidad de Santiago de Compostela \\ SPAIN
}

\begin{abstract}
The purpose of this paper is to study a nonlinear boundary value problem of second order when the nonlinearity is a Caratheodory function. It is shown that a generalized upper and lower solutions method is valid, and the monotone iterative technique for finding the minimal and maximal solutions is developed.
\end{abstract}

Key words: Periodic boundary value problem, upper and lower solutions, monotone method.

AMS (MOS) subject classification: 34B15.

\section{INTRODUCTION}

We shall, in this paper, develop the method of upper and lower solutions and the monotone iterative technique for second order boundary value problems of the form

$$
\begin{gathered}
-u^{\prime \prime}(t)=f(t, u(t)), t \in I=[0, \pi] \\
B u(0)=c_{0} \\
B u(\pi)=c_{1}
\end{gathered}
$$

where $f$ is a Carathéodory function, $B u(0)=a_{0} u(0)-b_{0} u^{\prime}(0)$, and $B u(\pi)=a_{1} u(\pi)+b_{1} u^{\prime}(\pi)$,

\footnotetext{
${ }^{1}$ Received: May, 1991. Revised: July, 1991.

${ }^{2}$ The authors were partially supported by DGICYT (project PS88-0054), and by Xunta de Galicia (project XUGA 20701A90), respectively.
} 
$a_{0}, a_{1} \geq 0, b_{0}, b_{1}>0$.

We first note that the classical arguments of [2] for $f$ continuous are no longer valid since if $u$ is a solution of $(P)$, then $u^{\prime \prime}$ needs not to be continuous but only $u^{\prime \prime} \in L^{1}(0, \pi)$. Here we extend classical and well-known results when $f$ is continuous (see [2]) to the case when $f$ is a Carathéodory function.

If we choose $a_{0}=a_{1}=c_{0}=c_{1}=0$, then the boundary conditions read $u^{\prime}(0)=u^{\prime}(\pi)=0$. Thus, we have the Neumann boundary value problem

$$
-u^{\prime \prime}=f(t, u), u^{\prime}(0)=u^{\prime}(\pi)=0 .
$$

We shall consider in Sections 2 and 3 this simpler boundary value problem so as to clearly bring out the ideas involved. On the other hand, there is no additional complication in studying $(P)$ instead of $(N)$. We list the corresponding results for $(P)$ in Section 4 .

Finally, in Section 5 and following the ideas developed in previous sections, we present the method of upper and lower solutions for the boundary value problem $(P)$ when $a_{0}, a_{1}>0$ and $b_{0}, b_{1} \geq 0$. In particular, we do so for the Dirichlet problem

$$
-u^{\prime}=f(t, u), u(0)=u(\pi)=0 .
$$

\section{GENERALIZED UPPER AND LOWER SOLUTIONS}

Let us assume that $f: I \times \mathbb{R} \rightarrow \mathbb{R}$ is a Caratheodory function, that is, $f(\cdot, u)$ is measurable for every $u \in \mathbb{R}$ and $f(t, \cdot)$ is continuous for a.e. $t \in I$. Moreover, we suppose that for every $R>0$ there exists a function $h=h_{R} \in L^{1}(I)$ with

$$
|f(t, u)| \leq h(t) \text { for a.e. } t \in I \text { and every }|u| \leq R .
$$

Let $E=\left\{u \in W^{2,1}(I): \quad u^{\prime}(0)=u^{\prime}(\pi)=0\right\}$ with the norm of $W^{2,1}(I)$ and $F=L^{1}(I)$ with the usual one. We shall denote by $\|\cdot\|_{E}$ and $\|\cdot\|$ the norms in $E$ and $F$, respectively. By a solution of $(N)$ we mean a function $u \in E$ satisfying the equation for a.e. $t \in I$.

Now, suppose that $\alpha, \beta \in W^{2,1}(I)$ are such that $\alpha(t) \leq \beta(t), t \in I$. Then, relative to $(N)$ we shall consider the following modified problem

$$
-u^{\prime \prime}(t)=g(t, u(t))-u(t)+p(t, u(t)), u^{\prime}(0)=u^{\prime}(\pi)=0,
$$

where 


$$
g(t, u)=f(t, p(t, u)) \text { and } \quad p(t, u)=\left\{\begin{array}{l}
\alpha(t) \text { for } u<\alpha(t) \\
u \text { for } \alpha(t) \leq u \leq \beta(t) \\
\beta(t) \text { for } u>\beta(t) .
\end{array}\right.
$$

We note that $g$ is a Carathéodory function and that the Neumann problem $(N)$ is equivalent to the integral equation

with

$$
u(t)=u(0)-\int_{0}^{t}(t-s) f(s, u(s)) d s
$$

$$
\int_{0}^{\pi} f(s, u(s)) d s=0
$$

We say that $\alpha \in W^{2,1}(I)$ is a lower solution for $(N)$ if

$$
-\alpha^{\prime \prime}(t) \leq f(t, \alpha(t)) \text { for a.e. } t \in I
$$

and

$$
\alpha^{\prime}(0) \geq 0 \geq \alpha^{\prime}(\pi)
$$

Similarly, $\beta \in W^{2,1}(I)$ is an upper solution for $(N)$ if

$$
-\beta^{\prime}(t) \geq f(t, \beta(t)) \text { for a.e. } t \in I
$$

and

$$
\beta^{\prime}(0) \leq 0 \leq \beta^{\prime}(\pi)
$$

We are now in a position to prove the following result which shows that the method of upper and lower solutions is still valid when $f$ is a Carathéodory function.

Theorem 2.1: $\quad$ Suppose that $\alpha, \beta \in W^{2,1}(I)$ are lower and upper solutions for $(N)$, respectively, such that $\alpha(t) \leq \beta(t)$ for every $t \in I$. Then there exists at least one solution $u$ of $(N)$ such that $\alpha(t) \leq u(t) \leq \beta(t)$ for every $t \in I$.

Proof: We first note that any solution $u$ of $(N)$ such that $\alpha \leq u \leq \beta$ is also a solution of (2.2). On the other hand, any solution $u$ of (2.2) with $\alpha \leq u \leq \beta$ is a solution of $(N)$. We shall show that any solution $u$ of (2.2) is such that $\alpha \leq u \leq \beta$ on $I$ and that (2.2) has at least one solution.

Now, let $u$ be a solution of (2.2). We first show that $\alpha(t) \leq u(t)$, for every $t \in I$. If $\alpha(t)>u(t)$ for every $t \in I$, then $-u^{\prime \prime}(t)=f(t, \alpha(t))-u(t)+\alpha(t)$ for a.e. $t \in I$. Thus we obtain the following contradiction 


$$
0=\int_{0}^{\pi} u^{\prime \prime}(t) d t=\int_{0}^{\pi}[f(t, \alpha(t))+\alpha(t)-u(t)] d t>-\int_{0}^{\pi} \alpha^{\prime \prime}(t) d t=\alpha^{\prime}(0)-\alpha^{\prime}(\pi) \geq 0 .
$$

Thus, there exists $t_{1} \in I$ with $\alpha\left(t_{1}\right) \leq u\left(t_{1}\right)$. Now, suppose that there exists $t^{\prime} \in I$ such that $\alpha\left(t^{\prime}\right)>u\left(t^{\prime}\right)$. Set $\varphi=\alpha-u$ and let $t_{0} \in I, \varphi\left(t_{0}\right)=\max \{\varphi(t): t \in I\}$. We first suppose that $t_{0} \in(0, \pi)$ and $t_{0}<t_{1}$ (the case $t_{0}>t_{1}$ is similar). Then $\varphi^{\prime}\left(t_{0}\right)=0$ and there exists $t_{2} \in\left(t_{0}, t_{1}\right)$ with $\varphi\left(t_{2}\right)=0$ and $\varphi(t)>0$ for every $t \in\left[t_{0}, t_{2}\right)$. On the other hand, we have that $\varphi^{\prime \prime}(t) \geq \varphi(t)>0$ for a.e. $t \in\left[t_{0}, t_{2}\right)$. This implies that $\varphi^{\prime}$ is increasing on $\left[t_{0}, t_{2}\right)$ and, in consequence, $\varphi^{\prime}(t) \geq 0, t \in\left[t_{0}, t_{2}\right)$ since $\varphi^{\prime}\left(t_{0}\right)=0$. Therefore, $\varphi$ is increasing on $\left[t_{0}, t_{2}\right)$ which is not possible.

Now, if $t_{0}=0$, then $\varphi^{\prime}(0) \leq 0$ and we get that $\varphi^{\prime}(0)=\alpha^{\prime}(0) \geq 0$ and $\varphi^{\prime}(0)=0$. As before, there exists $t_{2}>0$ such that $\varphi\left(t_{2}\right)=0$ and $\varphi(t)>0$ for every $t \in\left[0, t_{2}\right)$ and $\varphi^{\prime}$ is increasing on $\left[0, t_{2}\right)$ which contradicts that $\varphi\left(t_{2}\right)=0$. The case $t_{0}=\pi$ is analogous.

This shows that $\alpha(t) \leq u(t)$ for every $t \in I$ and by the same reasoning we obtain that $u(t) \leq \beta(t)$ for every $t \in I$.

We next prove that $(2.2)$ has at least one solution. For $\lambda \in[0,1]$, consider the following Neumann boundary value problem

$$
-u^{\prime \prime}(t)+u(t)=\lambda[g(t, u(t))+p(t, u(t))], u^{\prime}(0)=u^{\prime}(\pi)=0
$$

In order to apply the well-known theorem of Leray-Schauder, define the operators $L: E \rightarrow F$ and $N: F \rightarrow F$ by $L u=-u^{\prime \prime}+u$ and $N u=g(\cdot, u(\cdot))+p(\cdot, u(\cdot))$ respectively. Note that $L$ is continuous, one-to-one, and onto. Thus, the Neumann problem (2.9) is equivalent to the abstract equations

$$
L u=\lambda N u, \lambda \in[0,1], u \in E
$$

or

$$
u=\lambda H N u, \lambda \in[0,1], u \in F,
$$

where $H=i \cdot L^{-1}: F \rightarrow F$ and $i: E \rightarrow F$ is the canonical injection. $H$ is continuous and compact since $W^{2,1}(I)$ is compactly imbedded into $L^{1}(I)$.

Let $\gamma=\min \{\alpha(t): t \in I\}$ and $\delta=\max \{\beta(t): t \in I\}$. If $u$ is a solution of (2.2), then $|u(t)| \leq R=\max \{\gamma, \delta\}$ for every $t \in I$. Taking into account this, condition (2.1), and that $\alpha(t) \leq p(t, u(t)) \leq \beta(t)$ for every $t \in I$, we have 


$$
\|\lambda N u\| \leq\left\|h_{R}\right\|+2 \pi R=C .
$$

In consequence, if $u$ is a solution of (2.9) we have that $\|u\|_{E} \leq C \cdot\|H\|$, where $C$ is a constant independent of $\lambda \in[0,1]$ and $u \in F$. Thus, we have proved that all the solutions of (2.11) are bounded independent of $\lambda \in[0,1]$ and we can conclude that (2.11) with $\lambda=1$, that is (2.2), is solvable. This concludes the proof of the theorem.

\section{THE MONOTONE METHOD}

When $f$ is a continuous function the following comparison result is fundamental in the development of the monotone iterative technique.

Lemma 3.1: Let $\varphi \in C^{2}(I)$ and $\varphi^{\prime}(0) \geq 0 \geq \varphi^{\prime}(\pi)$. Suppose that there exists $M>0$ with $\varphi^{\prime \prime}(t) \geq M \varphi(t)$ for a.e. $t \in I$. Then $\varphi(t) \leq 0$ for every $t \in I$.

We now extend this result in order to cover the case when $f$ is a Caratheodory function.

Lemma 3.2: $\quad$ Let $\varphi \in W^{2,1}(I), \varphi^{\prime}(0) \geq 0 \geq \varphi^{\prime}(\pi)$. If there exists $M \in L^{1}(I)$ with $M(t)>0$ for a.e. $t \in I$ such that $\varphi^{\prime \prime}(t) \geq M(t) \varphi(t)$ for a.e. $t \in I$, then $\varphi(t) \leq 0$ for every $t \in I$.

Proof: If $\varphi(t)>0$ for every $t \in I$, then $\varphi^{\prime \prime}(t)>0$ for a.e. $t \in I$. Thus, $\varphi^{\prime}$ is strictly increasing on $I$ and $\varphi^{\prime}(0)<\varphi^{\prime}(\pi)$ which is a contradiction. Now, if there exists some $t \in I$ with $\varphi(t)>0$, then choose $s \in I$ such that $\varphi(s)=\max \{\varphi(t): t \in I\}$. If $s \in(0, \pi)$, then $\varphi^{\prime}(s)=0$ and there exists $t_{1} \in[0, s)$ (or $t_{1} \in(s, \pi]$ and the reasoning is analogous) with $\varphi\left(t_{1}\right)=0$ and $\varphi(t)>0$ for every $t \in\left(t_{1}, s\right)$. However, $\varphi^{\prime \prime}(t)>0$ for a.e. $t \in\left(t_{1}, s\right)$ and $\varphi^{\prime}$ is increasing on $\left(t_{1}, s\right)$. Hence, $\varphi^{\prime}(t) \leq 0$ for $t \in\left(t_{1}, s\right)$ and $\varphi$ is decreasing on $\left(t_{1}, s\right)$ which is not possible. If $s=0$ or $s=\pi$ the argument is similar. This completes the proof.

For $M \in F$ with $M(t)>0$ for a.e. $t \in I$ and $\eta \in F$ we shall consider the following Neumann boundary value problem

$$
-u^{\prime \prime}(t)=f(t, \eta(t))-M(t)[u(t)-\eta(t)], u^{\prime}(0)=0=u^{\prime}(\pi)
$$

or equivalently

$$
-u^{\prime \prime}(t)+M(t) u(t)=f(t, \eta(t))+M(t) \eta(t), u^{\prime}(0)=0=u^{\prime}(\pi) .
$$

The operator $L$ (defined in the proof of Theorem 2.1) is continuous, one-to-one and 
onto. Thus, by the open mapping theorem, its inverse $L^{-1}$ is continuous. For $\sigma \in F$, let $L^{-1} \sigma=u$ be the unique solution of the linear problem $-u^{\prime \prime}+M u=\sigma, u^{\prime}(0)=0=u^{\prime}(\pi)$.

If $\alpha, \beta$ are lower and upper solutions for $(N)$ respectively, let us introduce the following condition in order to develop the monotone method: There exists $M \in F$ with $M(t) \geq 0$ for a.e. $t \in I$ and we have that

$$
f(t, u)-f(t, v) \geq-M(t)(u-v)
$$

for a.e. $t \in I$ and for every $u, v \in \mathbb{R}$ such that $\alpha(t) \leq u \leq v \leq \beta(t)$.

For $\eta \in F$ with $\alpha \leq \eta \leq \beta$, that is, $\eta \in[\alpha, \beta]=\{u \in F: \alpha \leq u \leq \beta$ for a.e. $t \in I\}$, let us define the (nonlinear) operator $K:[\alpha, \beta] \rightarrow E$ by $K \eta=L^{-1} \sigma$ where $\sigma(t)=f(t, \eta(t))+M(t) \eta(t)$, $t \in I$. The operator $K$ is monotone and its properties are summarized in the following result.

Lemma 3.3: $\quad$ Assume that (3.3) holds. Then the operator $K$ has the following properties.

$$
\text { If } \alpha \leq \eta \leq \beta \text { on I, then } \alpha \leq K \eta \leq \beta \text { on I }
$$

and

$$
\text { if } \alpha \leq \eta_{1} \leq \eta_{2} \leq \beta \text { on } I \text {, then } \alpha \leq K \eta_{1} \leq K \eta_{2} \leq \beta \text { on } I \text {. }
$$

Proof: Let $\alpha \leq \eta \leq \beta$ on $I$. We shall prove that $u \leq \beta$ on $I$. Indeed, let $\varphi=u-\beta$. Thus, for a.e. $t \in I$ we have that

$$
\begin{aligned}
\varphi^{\prime \prime}(t) & =u^{\prime \prime}(t) \geq-f(t, \eta(t))-M(t) \eta(t)+M(t) u(t)+f(t, \beta(t)) \geq \\
& -M(t)[\beta(t)-\eta(t)]-M(t) \eta(t)+M(t) u(t)=M(t) \varphi(t) .
\end{aligned}
$$

By Lemma 3.1 we can conclude that $\varphi(t) \leq 0$ for every $t \in I$, that is, $u \leq \beta$ on $I$. The proof that $\alpha \leq u$ is similar.

To show that validity of (3.5), let $\varphi=K \eta_{1}-K \eta_{2}$. Thus, $\varphi^{\prime \prime}(t) \geq M(t) \varphi(t)$ for a.e. $t \in I$ and, in consequence, we obtain that $K \eta_{1} \leq K \eta_{2}$ on $I$.

Theorem 3.4: $\quad$ Suppose that $\alpha$ and $\beta$ are lower and upper solutions, respectively, of $(N)$ such that $\alpha \leq \beta$ on $I$ and (3.3) holds. Then, there exists monotone sequences $\left\{\alpha_{n}\right\} \uparrow$ and $\left\{\beta_{n}\right\} \downarrow$ with $\alpha_{0}=\alpha, \beta_{0}=\beta, \alpha_{n} \leq \beta_{m}$ for every $n, m \in N$ and $\lim _{n \rightarrow \infty} \alpha_{n}=r, \lim _{n \rightarrow \infty} \beta_{n}=\rho$ uniformly on $I$. Here, $r$ and $\rho$ are respectively the minimal and maximal solutions of $(N)$ between $\alpha$ and $\beta$ in the sense that if $u$ is a solution with $\alpha \leq u \leq \beta$ on $I$, then $r \leq u \leq \rho$ on $I$. 
Proof: Let $\alpha_{0}=\alpha$ and $\alpha_{n}=K \alpha_{n-1}(n=1,2, \ldots)$. We first prove that $\alpha_{0} \leq \alpha_{1}$. Indeed, let $\varphi=\alpha_{0}-\alpha_{1}$. Thus, $\quad \varphi^{\prime \prime}(t) \geq-f(t, \alpha(t))-M(t) \alpha_{1}(t)+f(t, \alpha(t))+$ $M(t) \alpha(t)=M(t) \varphi(t)$ for a.e. $t \in I$. This implies that $\varphi \leq 0$ on $I$ in view of Lemma 3.2. Taking into account property (3.5) we see that $\alpha_{1}=K \alpha_{0} \leq K \alpha_{1}=\alpha_{2}$ and, by induction, that $\alpha_{n} \leq \alpha_{n+1}$ for every $n \in N$. Similarly, defining $\beta_{0}=\beta$ and $\beta_{n}=K \beta_{n-1}$ we have that $\beta_{n+1} \leq \beta_{n}, n \in N$. Combining properties (3.4) and (3.5) we see that $\alpha \leq \alpha_{n} \leq \beta_{m} \leq \beta$ for every $n, m \in \mathbf{N}$.

Therefore, the sequence $\left\{\alpha_{n}\right\}$ is uniformly bounded and increasing and it has a pointwise limit, say $r(t), t \in I$. We now prove that $r$ is a solution of $(N)$. Choose $R>0$ such that $\left|\alpha_{n}(t)\right| \leq R$ for every $n \in \mathrm{N}, t \in I$. The sequence $\left\{\alpha_{n}^{\prime \prime}\right\}$ is bounded in $F$ since

$$
-\alpha_{n}^{\prime \prime}(t)=-M(t) \alpha_{n}(t)+f\left(t, \alpha_{n-1}(t)\right)+M(t) \alpha_{n-1}(t)
$$

and hence, $\quad\left\|\alpha_{n}^{\prime \prime}\right\| \leq\|M\| \cdot\left\|\alpha_{n}\right\|+\left\|h_{R}\right\|+\|M\| \cdot\left\|\alpha_{n-1}\right\| \leq 2\|M\| \cdot R \cdot 2 \pi+$ $\left\|h_{R}\right\|=C$. Here, $C$ is a constant independent of $n \in \mathbf{N}$.

On the other hand, $\alpha_{n}^{\prime}(t)=\int_{0}^{t} \alpha_{n}^{\prime \prime}(s) d s$, which implies that the sequence $\left\{\alpha_{n}^{\prime}\right\}$ is bounded in $L^{\infty}(I)$.

Therefore, $\left\{\alpha_{n}\right\}$ is bounded in $E$. This together with the montonicity of $\left\{\alpha_{n}\right\}$ implies that $\left\{\alpha_{n}\right\}$ is uniformly convergent to $r$. From (3.6) we obtain that

and

$$
\alpha_{n}(t)=\alpha_{n}(0)=\int_{0}^{t}(t-s)\left[M(s) \alpha_{n}(s)-f\left(s, \alpha_{n-1}(s)\right)-M(s) \alpha_{n-1}(s)\right] d s
$$

$$
\int_{0}^{\pi} f\left(s, \alpha_{n-1}(s)\right) d s=\int_{0}^{\pi} M(s)\left[\alpha_{n}(s)-\alpha_{n-1}(s)\right] d s .
$$

Letting $n \rightarrow \infty$ and using the uniform convergence of $\left\{\alpha_{n}\right\}$, we see that $r$ satisfies the integral equation (2.3) and (2.4), that is, $r$ is a solution of $(N)$.

Using the same integral representation for the solutions of $(N)$ we get that $\left\{\beta_{n}\right\}$ converges uniformly to a solution $\rho$ of $(N)$ and it is obvious that $\alpha \leq r \leq \rho \leq \beta$.

Finally, if $u$ is a solution of $(N)$ with $\alpha \leq u \leq \beta$ on $I$, then $\alpha_{1} \leq K u=u \leq \beta_{1}$. By induction we get that $\alpha_{n} \leq u \leq \beta_{n}$ for every $n \in \mathbb{N}$ which implies that $r \leq u \leq \rho$ and concludes the proof of the theorem. 


\section{NONLINEAR SECOND ORDER BOUNDARY VALUE PROBLEMS}

A function $v \in W^{2,1}(I)$ is said to be a lower solution of $(P)$ if

$$
-v^{\prime \prime}(t) \leq f(t, v(t)) \text { for a.e. } t \in I
$$

and

$$
B v(0) \leq c_{0}, B v(\pi) \leq c_{1},
$$

and an upper solution of $(P)$ if the reversed inequalities hold in (4.1) and (4.2).

If we know the existence of upper and lower solutions for $(P)$, then we can guarantee the existence of a solution for $(P)$.

Theorem 4.1: Assume that $\alpha$ and $\beta$ are lower and upper solutions of $(P)$ respectively, such that $\alpha(t) \leq \beta(t)$ for every $t \in I$. Then there exists at least one solution $u$ for the problem $(P)$ such that $\alpha(t) \leq u(t) \leq \beta(t), t \in I$.

In order to develop the monotone method, we need the following result which is analogous to Lemma 3.2.

Lemma 4.2: Let $\varphi \in W^{2,1}(I)$ be such that $B \varphi(0) \leq 0$ and $B \varphi(\pi) \leq 0$. Assume that there exists $M \in L^{1}(I)$ such that $M(t)>0$ for a.e. $t \in I$, and $\varphi^{\prime \prime}(t) \geq M(t) \varphi(t)$ for a.e. $t \in I$. Then $\varphi(t) \leq 0$ for every $t \in I$.

Proof: If $\varphi(t)>0$ for every $t \in I$, then $\varphi^{\prime \prime}(t)>0$ for a.e. $t \in I$ and $\varphi^{\prime}$ is strictly increasing on $I$ and $\varphi^{\prime}(0)<\varphi^{\prime}(\pi)$. However, $B \varphi(0) \leq 0$ and $B \varphi(\pi) \leq 0$ implies that $\varphi^{\prime}(0) \geq 0$ and $\varphi^{\prime}(\pi) \leq 0$ which is a contradiction. Now, reasoning as in the proof of Lemma 3.2 we see that there is no $t \in I$ with $\varphi(t)>0$.

This allows us to show the validity of the monotone iterative technique for the boundary value problem $(P)$.

Theorem 4.3: Let the assumptions of Theorem 4.1 hold. In addition, suppose that there exists $M \in L^{1}(I)$ and $M(t)>0$ for a.e. $t \in I$, such that for a.e. $t \in I$ and every $u, v \in \mathbb{R}$ with $\alpha(t) \leq u \leq v \leq \beta(t)$ we have

$$
f(t, u)-f(t, v) \geq-M(t)(u-v) .
$$

Then there exist monotone sequences $\left\{\alpha_{n}\right\} \nmid r$ and $\left\{\beta_{n}\right\} \downarrow \rho$ uniformly on $I$. Here, $r$ and $\rho$ are the minimal and maximal solutions respectively, of $(P)$ between $\alpha$ and $\beta$.

Proof: $\quad$ For $\alpha \leq \eta \leq \beta$, we solve the boundary value problem 


$$
-u^{\prime \prime}(t)+M(t) u(t)=f(t, \eta(t))+M(t) \eta(t), B u(0)=c_{0}, B u(\pi)=c_{1}
$$

which has a unique solution $u=K \eta$.

The operator $K$ has the properties (3.4) and (3.5) and then one can generate the monotone iterates.

\section{DIRICHLET PROBLEM}

We say that $\alpha \in W^{2,1}(I)$ is a lower solution of $(D)$ if $-\alpha^{\prime \prime}(t) \leq f(t, \alpha(t))$ for a.e. $t \in I, \alpha(0) \leq 0$, and $\alpha(\pi) \leq 0$. Similarly, $\beta$ is an upper solution if the reversed inequalities hold.

Now, using the following result it is easy to show that the monotone method for the Dirichlet problem $(D)$ is also valid.

Lemma 5.1: $\quad$ Let $\varphi \in W^{2,1}(I)$ and suppose that there exists $M \in L^{1}(I), M(t)>0$ for a.e. $t \in I$, such that $\varphi^{\prime \prime}(t) \geq M(t) \varphi(t)$ for a.e. $t \in I$. If $\varphi(0) \leq 0$ and $\varphi(\pi) \leq 0$, then $\varphi(t) \leq 0$ for every $t \in I$.

\section{REFERENCES}

[1] A. Cabada and J.J. Nieto, "A generalization of the monotone iterative technique for nonlinear second order periodic boundary value problems", J. Math. Anal. Appl. 151, (1990), pp. 181-189.

[2] G.S. Ladde, V. Lakshmikantham, and A.S. Vatsala, “Monotone Iterative Techniques for Nonlinear Differential Equations”, Pitman Publishing Inc., Boston (1985).

[3] M.N. Nkashama, "A generalized upper and lower solutions method and multiplicity results for nonlinear first order ordinary differential equations", J. Math. Anal. Appl. 140, (1989), pp. 381-395.

[4] J.J. Nieto, "Nonlinear second order periodic boundary value problems", J. Math. Anal. Appl. 130, (1988), pp. 22-29.

[5] J.J. Nieto, "Nonlinear second order periodic boundary value problems with Carathéodory functions", Applicable Anal. 34, (1989), pp. 111-128. 


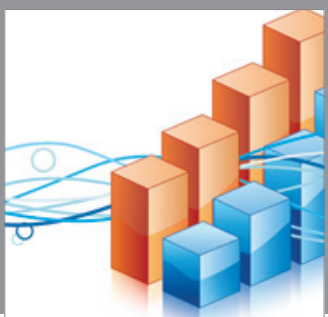

Advances in

Operations Research

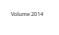

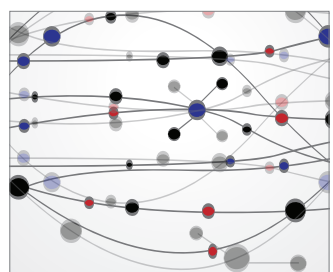

\section{The Scientific} World Journal
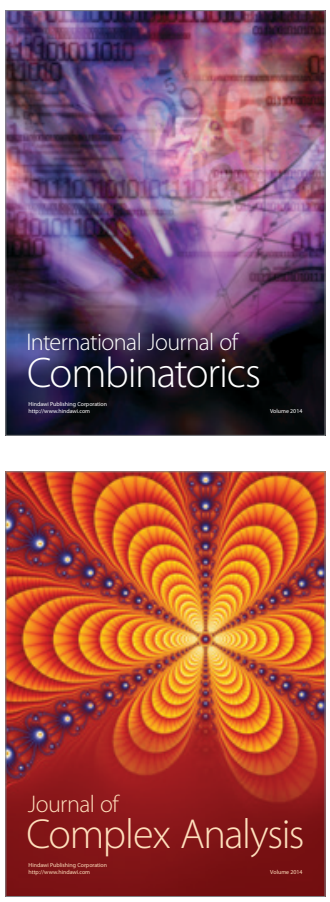

International Journal of

Mathematics and

Mathematical

Sciences
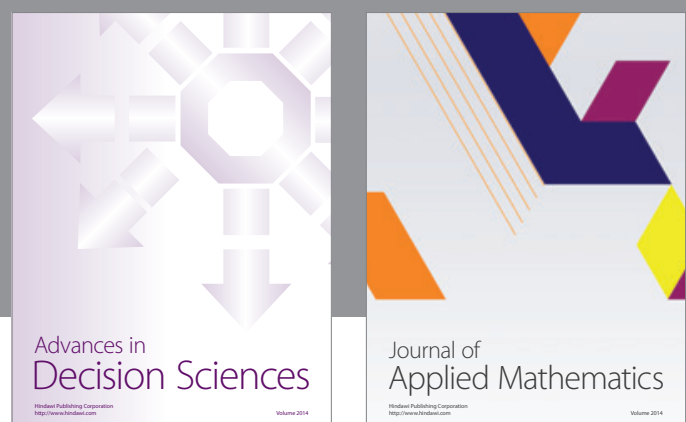

Journal of

Applied Mathematics
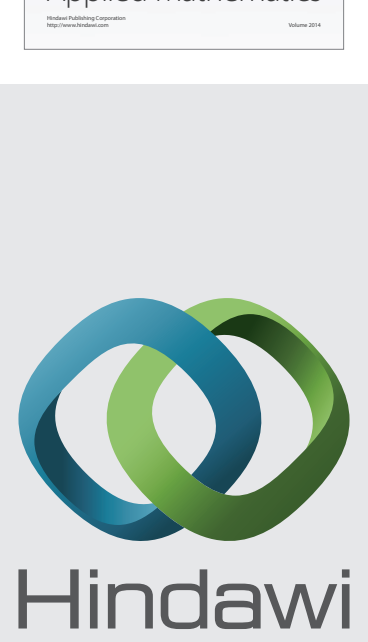

Submit your manuscripts at http://www.hindawi.com
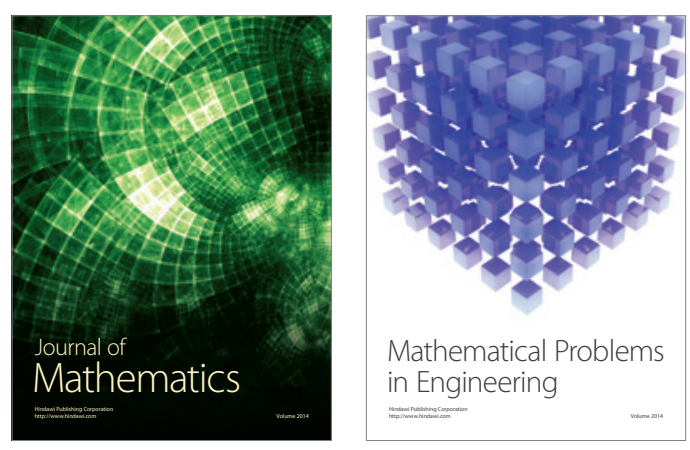

Mathematical Problems in Engineering
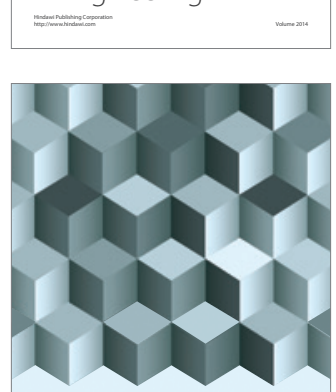

Journal of

Function Spaces
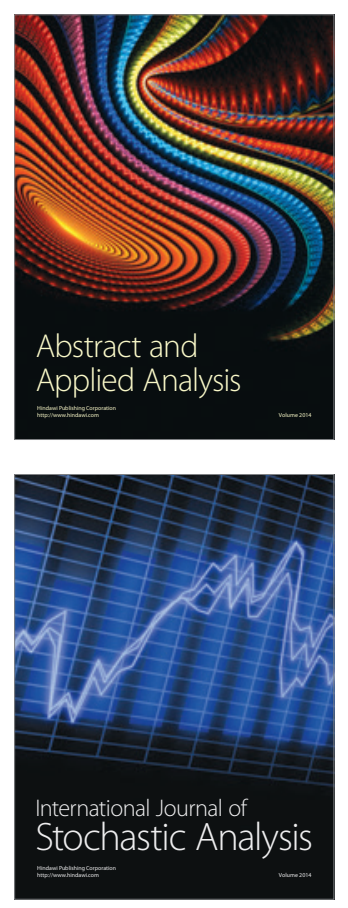

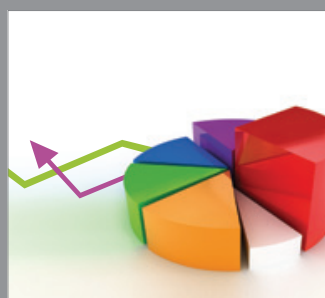

ournal of

Probability and Statistics

Promensencen
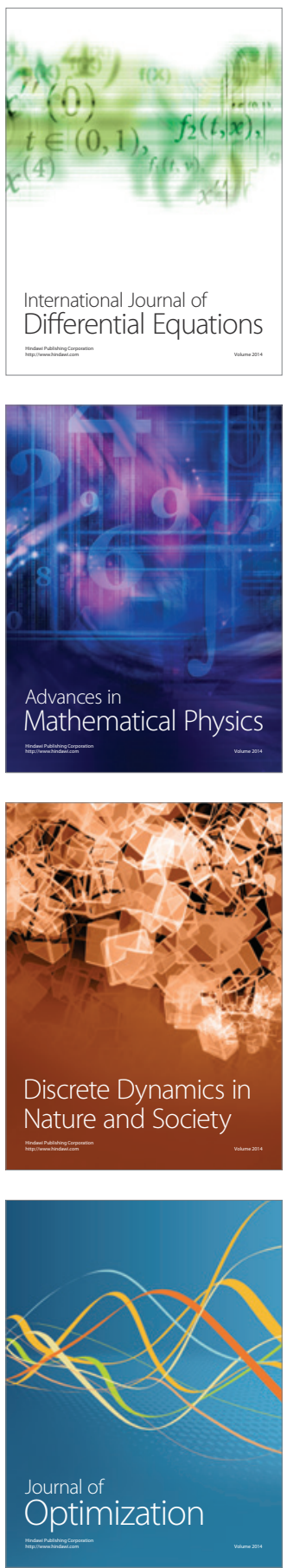